\title{
RSK3/4 mediate resistance to PI3K pathway inhibitors in breast cancer
}

\author{
Violeta Serra, ${ }^{1}$ Pieter J.A. Eichhorn, ${ }^{2}$ Celina García-García, ${ }^{1}$ Yasir H. Ibrahim, ${ }^{1}$ Ludmila Prudkin, ${ }^{3}$ \\ Gertrudis Sánchez,, ${ }^{3}$ Olga Rodríguez, ${ }^{1}$ Pilar Antón, ${ }^{1}$ Josep-Lluís Parra, ${ }^{4}$ Sara Marlow, ${ }^{5}$ \\ Maurizio Scaltriti, ${ }^{6}$ José Pérez-Garcia, ${ }^{7}$ Aleix Prat, ${ }^{8}$ Joaquín Arribas, ${ }^{4}$ William C. Hahn, ${ }^{9,10}$ \\ So Young Kim, ${ }^{11}$ and José Baselga ${ }^{1,6}$
}

\begin{abstract}
'Experimental Therapeutics, Vall d'Hebron Institute of Oncology, Barcelona, Spain. ${ }^{2}$ Cancer Science Institute of Singapore, National University of Singapore, Singapore. ${ }^{3}$ Molecular Pathology and ${ }^{4}$ Growth Factors Groups, Vall d'Hebron Institute of Oncology, Barcelona, Spain. ${ }_{5}^{5}$ Massachusetts General Hospital Cancer Center, Massachusetts General Hospital, Boston, Massachusetts, USA. ${ }^{6}$ Human Oncology and Pathogenesis Program and Memorial Sloan-Kettering Cancer Center, New York, New York, USA. 'BBreast Cancer and Melanoma Group and ${ }^{8}$ Translational Genomics Unit, Vall d'Hebron Institute of Oncology, Barcelona, Spain. ${ }^{9}$ Center for Cancer Genome Discovery, Department of Medical Oncology, Dana-Farber Cancer Institute and Harvard Medical School, Boston, Massachusetts, USA. ${ }^{10 B}$ road Institute of Harvard and MIT, Cambridge, Massachusetts, USA.

${ }^{11}$ Department of Molecular Genetics and Microbiology, Duke RNAi Screening Facility, Duke University Medical Center, Durham, North Carolina, USA.
\end{abstract}

\begin{abstract}
The PI3K signaling pathway regulates diverse cellular processes, including proliferation, survival, and metabolism, and is aberrantly activated in human cancer. As such, numerous compounds targeting the PI3K pathway are currently being clinically evaluated for the treatment of cancer, and several have shown some early indications of efficacy in breast cancer. However, resistance against these agents, both de novo and acquired, may ultimately limit the efficacy of these compounds. Here, we have taken a systematic functional approach to uncovering potential mechanisms of resistance to PI3K inhibitors and have identified several genes whose expression promotes survival under conditions of PI3K/mammalian target of rapamycin (PI3K/mTOR) blockade, including the ribosomal S6 kinases RPS6KA2 (RSK3) and RPS6KA6 (RSK4). We demonstrate that overexpression of RSK3 or RSK4 supports proliferation upon PI3K inhibition both in vitro and in vivo, in part through the attenuation of the apoptotic response and upregulation of protein translation. Notably, the addition of MEK- or RSK-specific inhibitors can overcome these resistance phenotypes, both in breast cancer cell lines and patient-derived xenograft models with elevated levels of RSK activity. These observations provide a strong rationale for the combined use of RSK and PI3K pathway inhibitors to elicit favorable responses in breast cancer patients with activated RSK.
\end{abstract}

\section{Introduction}

The PI3Ks, PKB/AKT, and mammalian target of rapamycin (mTOR) axis is integral for various physiological processes, including proliferation, growth, survival, and metabolism. Mutations of several components of the PI3K pathway that lead to constitutive activation of this pathway are found in human cancer. In particular, members of the class $\mathrm{I}_{\mathrm{A}}$ PI3K family, which are heterodimers comprising a p85 regulatory and a p110 catalytic subunit, are frequently mutated in solid tumor types, including breast, lung, ovarian, prostate, colorectal, and pancreatic cancers (1-3). Another frequent alteration leading to activation of PI3K signaling in human cancers is the inactivation of the phosphatase and tensin homolog $(P T E N)$ tumor suppressor through somatic mutations that result in protein truncation, homozygous or hemizygous deletions, or epigenetic silencing $(4,5)$. Additionally, other commonly mutated and/or amplified genes are upstream regulators of the PI3K pathway, including EGFR, HER2, IGFR, MET, and RAS,

Authorship note: Violeta Serra and Pieter J.A. Eichhorn contributed equally to this work.

Conflict of interest: José Baselga and William C. Hahn consult for Novartis Pharmaceuticals.

Note regarding evaluation of this manuscript: Manuscripts authored by scientists associated with Duke University, The University of North Carolina at Chapel Hill, Duke-NUS, and the Sanford-Burnham Medical Research Institute are handled not by members of the editorial board but rather by the science editors, who consult with selected external editors and reviewers.

Citation for this article: J Clin Invest. 2013;123(6):2551-2563. doi:10.1172/JCI66343 and are known to promote tumorigenicity, at least in part through the upregulation of PI3K signaling $(1,2)$.

Due to the importance of PI3K pathway activation in human cancer, several small molecule inhibitors targeting the PI3K/AKT/ mTOR pathway are currently under clinical development for treatment of cancer. The macrolide rapamycin and its analogs, such as RAD001 (everolimus), specifically inhibit mTORC1 and have profound cytostatic activity in preclinical models. Everolimus has been shown to provide clinical benefit in treatment of advanced renal cell carcinoma (6), neuroendocrine pancreatic tumors (7), and most recently, in hormone receptor-positive breast cancer, where it significantly delays disease progression when given in combination with hormonal therapy (8). Several recent reports have also demonstrated activity of PI3K inhibitors in preclinical models in specific subsets of breast cancer cells, including most notably with PI3K inhibitor monotherapy in PIK3CA-mutated and ERBB2-amplified breast cancers (9-11). In addition, clinical activity in patients with breast cancer harboring PIK3CA mutations has also been recently reported (12). However, experience with previous targeted therapy paradigms suggests that primary and acquired resistance will be a limiting factor with these agents. Therefore, a clear understanding of the mechanisms underlying PI3K inhibitor sensitivity and/or resistance will be invaluable in determining which patients are most likely to benefit. Moreover, identification of accurate biomarkers in patients who are unlikely to respond to PI3K inhibitor therapy may promote the development of rational drug combinations that will overcome 
A
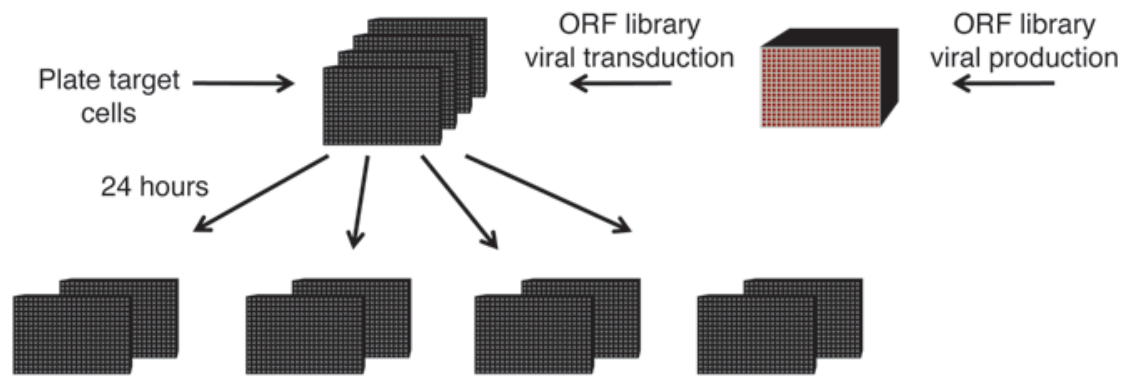

Non-selected

Blast-selected

BEZ235

BKM120

Assay for cell proliferation 5 days after drug addition
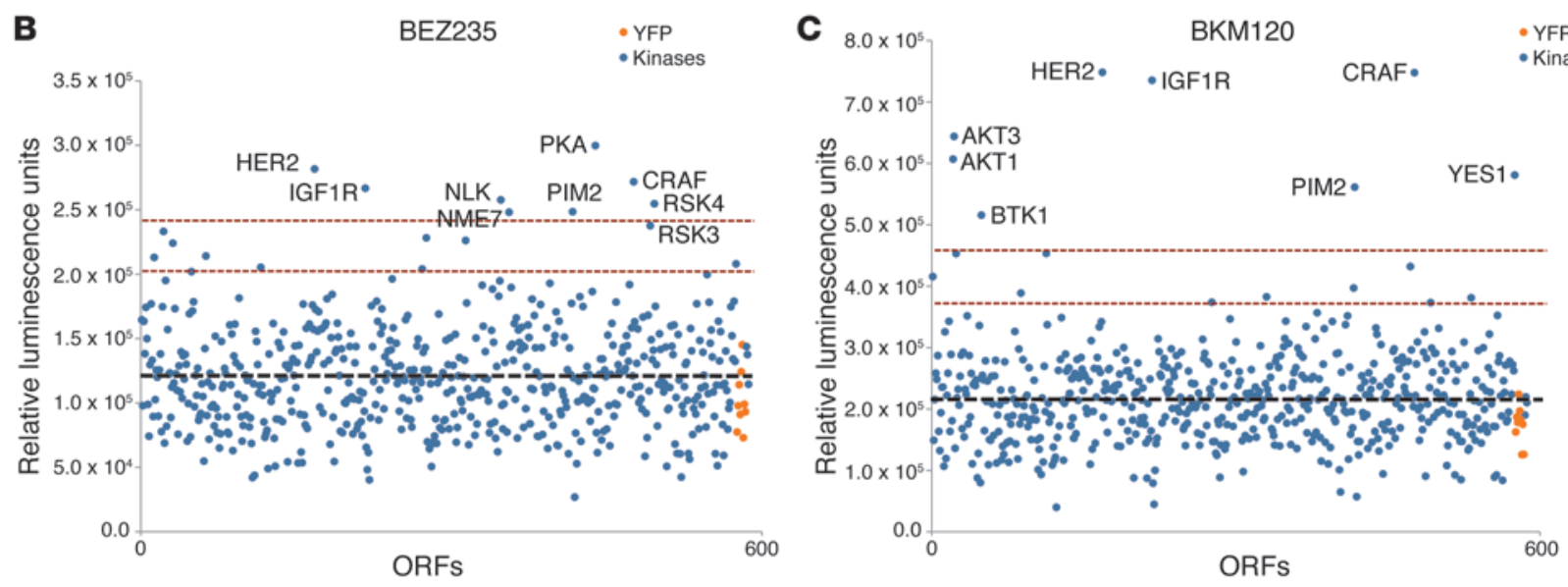

\section{Figure 1}

Overview of ORF kinase screen. (A) Schematic overview of screening process of lentivirally delivered ORF kinase library. MCF7 cells were transduced with kinase ORFs, treated with blasticidin, $200 \mathrm{nM} \mathrm{BEZ235,} \mathrm{or} 1 \mu \mathrm{M}$ BKM120, or left untreated for 5 days and then assayed for viability after 5 days. (B) Scatter plot of results of BEZ235 screen, with 2 and 3 SD (red lines) and mean (black line). Candidate hits were defined as kinases at least 3 SD above the mean. Orange circles represent YFP-infected control cells. (C) Results of BKM120 screen.

this problem. Recently, a number of clinical and preclinical studies have shown that enhanced ERK signaling, either by activation of compensatory feedback loops or intrinsic KRAS mutations, limits the effectiveness of PI3K pathway inhibitors (13-20). Also, MYC amplification, hyperactivation of the WNT/ $\beta$-catenin pathway, activation of NOTCH1, and amplification of the translation initiation factor eIF4E all appear able to promote PI3K inhibitor resistance to varying degrees (21-24). Here, using a systematic functional genetic screening approach, we have identified several kinases that mediate resistance to PI3K inhibition, including ribosomal S6 kinases RPS6KA2 (RSK3) and RPS6KA6 (RSK4).

RSK3 and RSK4 are members of the p90RSK family. RSKs are directly regulated by ERK signaling and are implicated in cell growth, survival, motility, and senescence (25-28). Here, we present evidence that overexpression of RSK3 and RSK4 supports cellular proliferation under PI3K pathway blockade by inhibiting apoptosis and regulating cellular translation through phosphorylation of ribosomal proteins S6 and eIF4B. We found RSK3 and RSK4 were overexpressed or activated in a fraction of breast cancer tumors and cell lines, supporting a role for these proteins in breast tumorigenesis. Furthermore, in 2 triple-negative breast cancer patient-derived primary tumor xenografts (PDX), we observed that the PDX with higher levels of phosphorylated RSK was resistant to PI3K inhibition. Importantly, we also demonstrate that by combining inhibitors of PI3K with inhibitors of MEK or RSK, we can reverse the resistance phenotype exhibited by breast cancer cell lines and PDX models with activated RSK and propose that this therapeutic combination may be clinically effective in patients with RSK-activated breast cancers.

\section{Results}

Kinase ORF expression screen. To identify kinases whose expression can mediate resistance to PI3K inhibitors, we performed open reading frame (ORF) expression screens in breast cancer cell lines in the presence of BEZ235 (dual PI3K/mTOR inhibitor) (29) or BKM120 (pan-PI3K inhibitor) (Figure 1). Both of these compounds are currently in clinical development $(30,31)$. This ORF library is composed of 597 kinases and kinase-related genes in lentiviral expression vectors containing a blasticidin resistance marker for efficient transduction and stable overexpression in target cells (32-34). We chose to perform a focused screen with kinases, as they represent a set of readily druggable targets, facilitating validation and potentially clinical translation. We screened MCF7 and BT474 cells, as they represent the 2 genotypes of breast cancer cells previously established as exhibiting sensitivity to PI3K inhibition, MCF7 (mutant PIK3CA) and BT474 (ERBB2 amplified) (9-11).

The criteria used to select kinases that support proliferation following PI3K/mTOR blockade in the ORF screen were (a) 

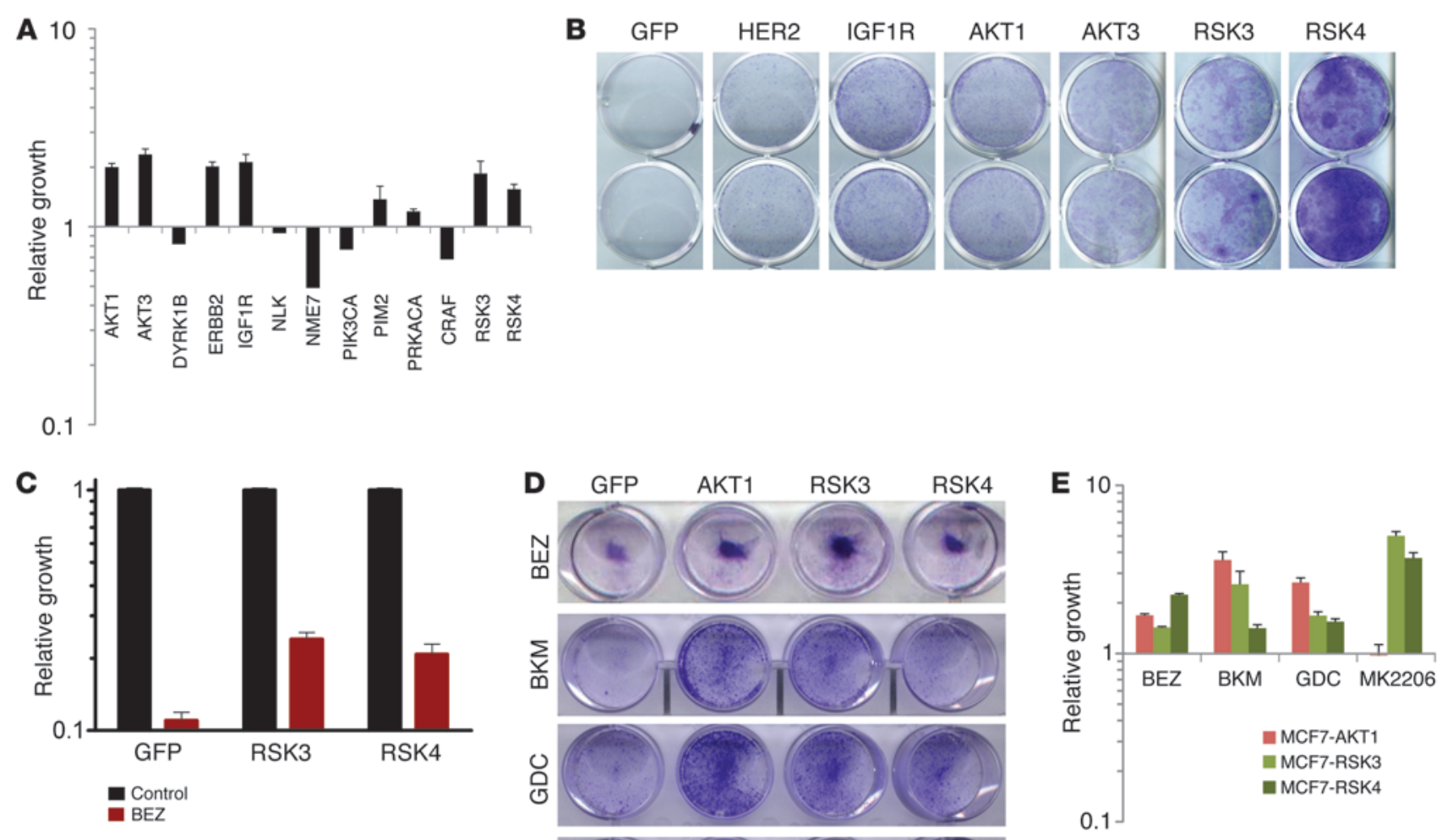

\section{Figure 2}

Validation of candidates from ORF screen. (A) Validation of top candidates from ORF kinase screen treated with BEZ235 (100 nM). Cells were assayed by CellTiter-Glo 5 days after drug addition. Bars represent fold increase relative to treated controls (mean \pm SD of representative experiment of 3 independent experiments). (B) Colony formation assay of MCF7 cells stably transduced with indicated ORF kinases and treated with $100 \mathrm{nM}$ BEZ235 for 14 days. (C) Cells were assayed by CellTiter-Glo 5 days after drug addition. Bars represent relative proliferation compared with untreated controls (mean \pm SD of 3 independent experiments). (D) Colony formation assay of AKT1-, RSK3-, and RSK4-overexpressing MCF7 cells treated with BEZ235 (100 nM), BKM120 (750 nM), GDC-0941 (1 $\mu \mathrm{M})$, and MK-2206 (2 $\mu \mathrm{M})$ for 8 days. (E) Quantification of crystal violet staining of RSK4-overexpressing MCF7 cells treated with BEZ235 (200 nM), BKM120 (750 nM), GDC-0941 (1 $\mu$ M), and MK-2206 (2 $\mu$ M) for 8 days. Bars represent fold increase relative to treated GFP-expressing controls (mean \pm SD of 3 independent experiments).

increased cell numbers in the presence of BEZ235 or BKM120 by at least $3 \mathrm{SD}$ above the mean and (b) corresponding increases in the ratio of cell number in treated versus untreated wells to remove kinases that simply stimulate general proliferation (Figure 1, Supplemental Table 1, and Supplemental Figure 1A; supplemental material available online with this article; doi:10.1172/JCI66343DS1). We performed validation experiments on the ORFs with the strongest phenotypes in the MCF7 screens for resistance against BEZ235 and BKM120 and were able to confirm PI3K inhibitor resistance phenotype for most of these candidates using 2 independent assays for viability (Figure 2, A and B, and Supplemental Figure 1B). Unsurprisingly, validated candidates included the receptor tyrosine kinases ERBB2 (HER2) and IGF1R, both of which are known to be upstream of PI3K-dependent signaling and PI3K-independent signaling as well as AKT1 and AKT3, key effectors of the PI3K pathway. Of the remaining candidates, we were particularly interested in RPS6KA2 (RSK3) and RPS6KA6 (RSK4), as these 2 genes provided robust resistance against PI3K inhibition (Figure $2 \mathrm{C}$ and Supplemental Figure 2A).
RSKs mediate resistance to PI3K inbibition. Since RSK3- and RSK4-overexpressing cells exhibited a profound decrease in PI3K inhibitor sensitivity, we sought to determine whether other RSK family members exhibited similar properties. In contrast to RSK3 and RSK4, expression of RSK1 and RSK2 only slightly decreased the sensitivity to PI3K inhibition, while the highly related mitogen- and stress-activated protein kinases (MSKs) exhibited no activity, and this was irrespective of expression levels (Supplemental Figure 2B). We therefore chose to focus on RSK3 and RSK4 for subsequent analyses.

To determine whether the resistance phenotypes of RSK-overexpressing cell lines extended to other PI3K pathway inhibitors, we determined the sensitivity of these cells to other inhibitors currently in early stage clinical testing, including GDC-0941, a pan PI3K inhibitor, and MK-2206, an allosteric pan-AKT inhibitor. As expected, treatment with all PI3K pathway inhibitors completely inhibited the proliferation potential of GFP-expressing control cells. However, RSK3 and RSK4 overexpression in MCF7 cells counteracted the growth inhibitory properties of all PI3K pathway inhibitors tested (Figure 2, D and E). In contrast, while 
A
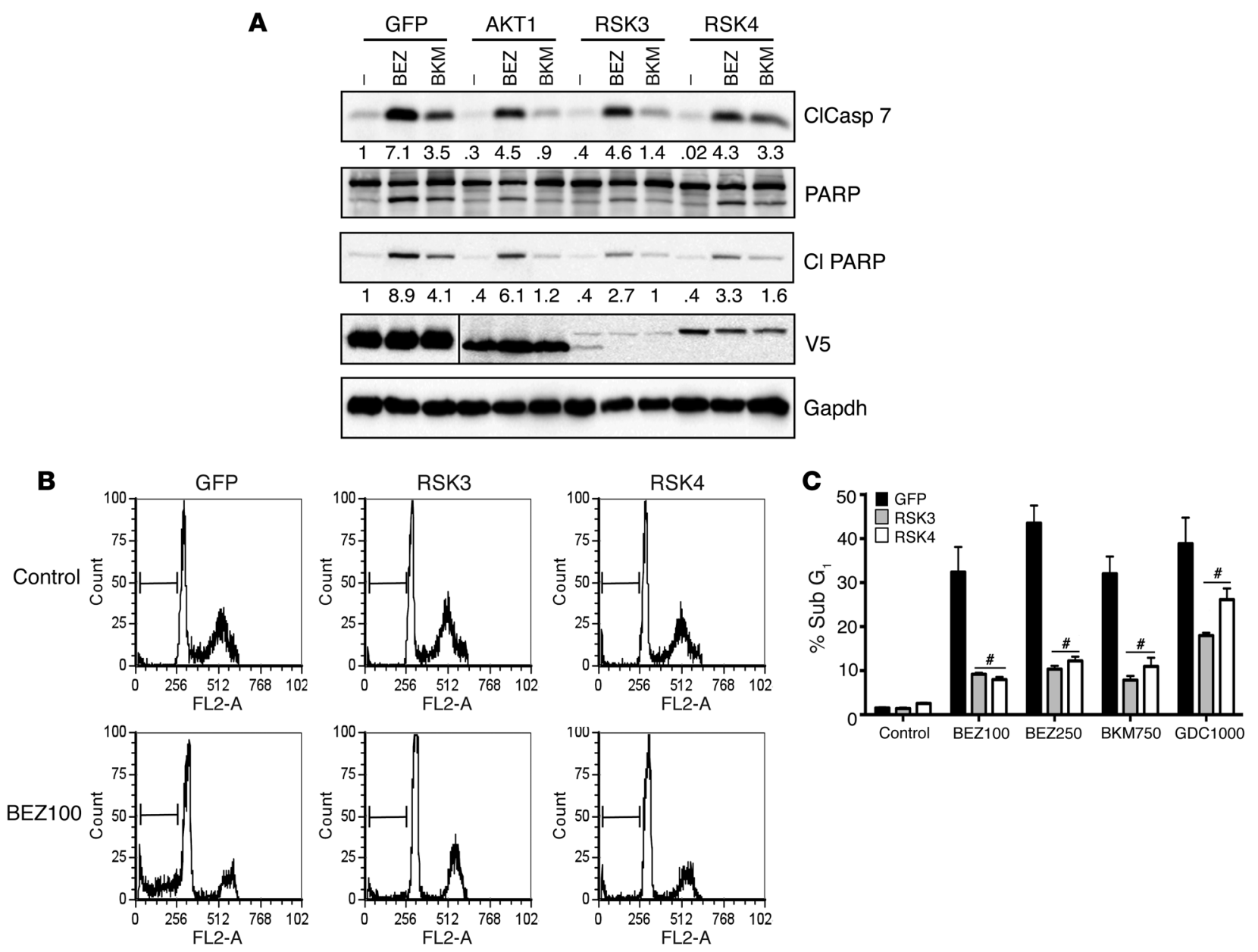

Figure 3

Reduced induction of apoptosis by PI3K inhibitors in RSK-overexpressing cells. (A) MCF7 cells expressing GFP, AKT1, RSK3, or RSK4 were treated with BEZ235 (100 nM) or BKM120 $(1 \mu \mathrm{M})$ for 24 hours. Also shown are band intensities of cleaved caspase 7 and cleaved PARP relative to untreated GFP control. V5-tagged proteins were run on the same blot, but bands were noncontiguous due to differences in protein size. (B) Cell-cycle analysis of MCF7 cells expressing GFP, RSK3, or RSK4 after 24 hours of treatment with BEZ235 (100 nM). (C) Quantification of sub$\mathrm{G}_{1}$ population after treatment with PI3K inhibitors as indicated, mean \pm SEM of 3 independent experiments. A 2-tailed Student's $t$ test compares the treated populations, ${ }^{\#} P<0.001$.

AKT1-expressing cells were resistant to the PI3K/mTOR-targeted agents, they remained sensitive to treatment with the AKT inhibitor MK2206 (Figure 2, D and E).

The RSK family of proteins comprises a group of highly related serine/threonine kinases that regulate cell growth, survival, and cellular proliferation downstream of the RAS/RAF/MEK/ERK pathway. To elucidate the mechanisms behind PI3K inhibitor resistance in RSK-overexpressing cells, we sought to uncover differences in cellular responses to PI3K/mTOR inhibition between control and RSK-overexpressing cells. Previous studies have established that BEZ235 induces apoptosis in cell lines sensitive to PI3K/ mTOR inhibition $(11,35)$. Since both RSK and AKT overexpression lead to decreased sensitivity to PI3K inhibitors, we reasoned that these attenuated responses might be due to the inhibition of apoptosis. As expected, the addition of either BEZ235 or BKM120 substantially enhanced PARP and caspase 7 cleavage, indicative of apoptosis, in GFP-expressing control cells. In contrast, we observed reduced cleaved PARP and cleaved caspase 7 in RSK3/4-
Vor AKT1-overexpressing cells upon treatment with BEZ235 or BKM120 (Figure 3A). Furthermore, treatment of control cells with BEZ235 led to increased PARP cleavage in a dose-dependent manner, which was again attenuated in cells expressing RSK or AKT1 (Supplemental Figure 2C). We also observed a marked decrease in the accumulation of cells in sub- $\mathrm{G}_{1}$ in the RSK4-overexpressing cells compared with control cells upon treatment with BEZ235 (Figure 3, B and C, and Supplemental Figure 3). Similar findings were observed in RSK-overexpressing cells treated with the panPI3K inhibitors BKM120 and GDC0941 (Figure 3C and Supplemental Figure 3). Taken together, these data suggest that RSK-overexpressing cells are resistant to PI3K/mTOR inhibition at least in part through decreased induction of apoptosis.

A number of recent reports have demonstrated that the antitumor effects of PI3K inhibition may be reduced by the activation of the ERK signaling pathway or by upregulation of protein translation $(22,24,36-38)$. Likewise, we investigated the regulation of protein translation in our RSK- or AKT1-overexpressing cells. 

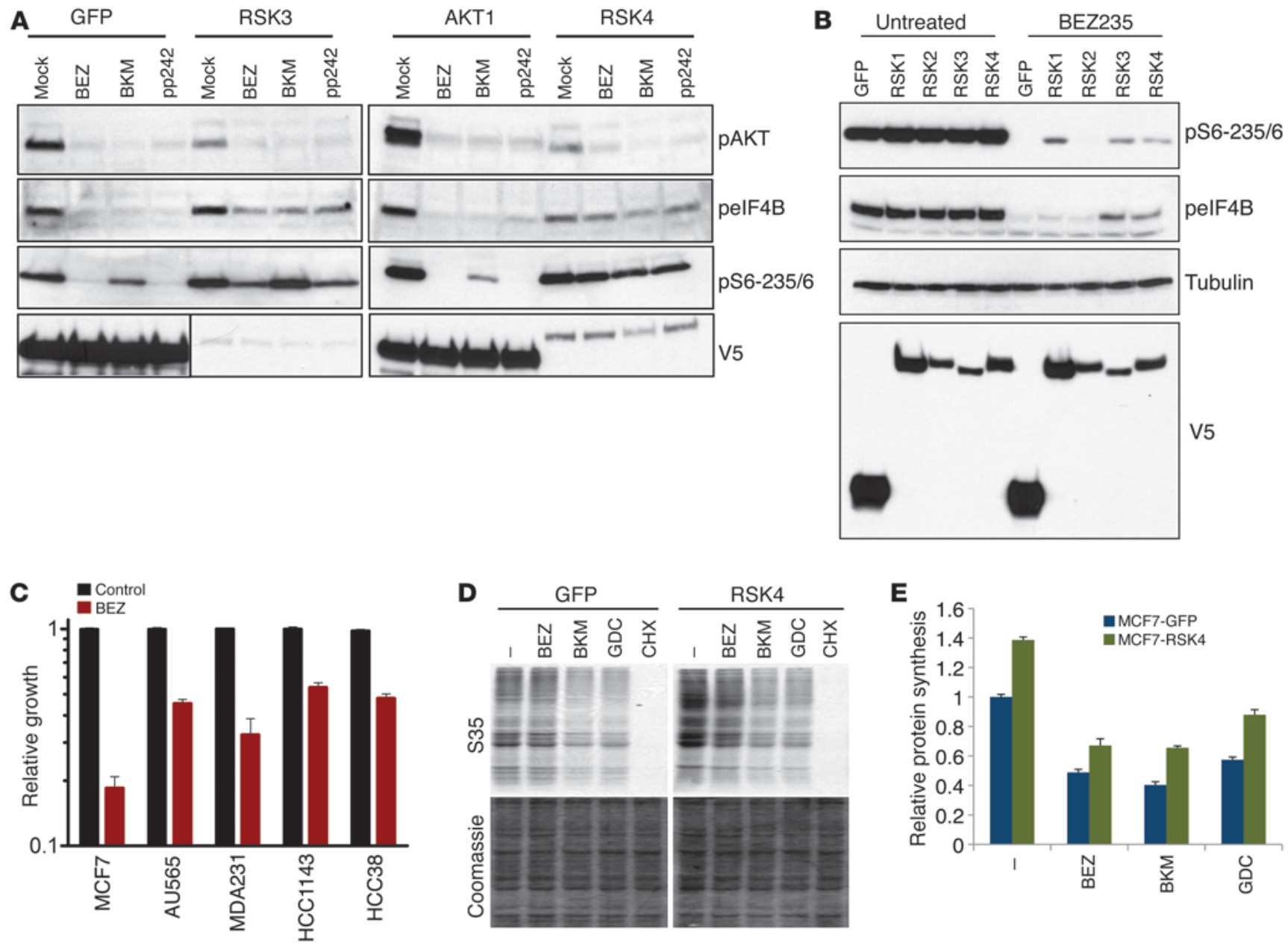

Figure 4

Reduced suppression of translation by PI3K inhibitors in RSK-overexpressing cells. (A) MCF7 cells stably expressing GFP, AKT1, RSK3, or RSK4 were treated with BEZ235 (100 nM), BKM120 $(1 \mu \mathrm{M})$, or pp242 $(1 \mu \mathrm{M})$ for 24 hours. The levels of the indicated proteins were determined by immunoblotting. V5-tagged proteins were run on the same blot, but bands were noncontiguous due to differences in protein size. (B) MCF7 cells overexpressing RSK1, RSK2, RSK3, and RSK4 were treated with BEZ235 (100 nM) for 24 hours. The levels of the indicated proteins were determined by immunoblotting. (C) Proliferation of breast cancer cells treated with BEZ235 (100 nM) for 24 hours, assessed by CellTiter-Glo. Bars represent relative proliferation compared with untreated controls (mean \pm SD of 3 independent experiments). (D) MCF7 cells overexpressing RSK4 were treated for 24 hours with indicated PI3K inhibitors prior to labeling new protein synthesis with ${ }^{35}$ S. Newly synthesized proteins were separated by SDS-PAGE, transferred to nitrocellulose, and visualized by autoradiography. (E) Quantification of newly synthesized protein (mean \pm SD of 3 independent experiments).

In control cells, PI3K pathway blockade with the PI3K inhibitor BKM120, the dual PI3K/mTOR inhibitor BEZ235, or the catalytic mTOR inhibitor pp242 (39) markedly reduced eIF4B and rpS6 phosphorylation, 2 key regulators of cap-dependent translation (Figure 4A). In contrast, dephosphorylation of ribosomal protein S6 and eIF4B by PI3K, mTOR, or dual PI3K/mTOR inhibitors was abrogated in the RSK-overexpressing cells (Figure 4A). We extended these analyses to other RSK family members. Although phospho-rpS6 was maintained in RSK1, RSK3, and RSK4 overexpressing cells, phospho-eIF4B was only detectable in RSK3- and RSK4-overexpressing cells following PI3K inhibition (Figure 4B). These results are in line with our proliferation studies suggesting that, while RSK1, RSK3 and RSK4 decrease the sensitivity of cells to PI3K inhibitors, only RSK3- and RSK4-overexpressing cells exhibit a strong resistance phenotype (Supplemental Figure 2B).
Two classes of protein kinases are known to phosphorylate rpS6 directly. The kinases primarily responsible for rpS6 phosphorylation are the mTOR-regulated $\mathrm{S} 6$ kinases, which are highly sensitive to PI3K/mTOR inhibition (40). The second class is the RSK family of kinases, which are regulated by ERK signaling and are activated following mitogenic stimulation (41). Based on our observation that retention of $\mathrm{rpS} 6$ and $\mathrm{IF} 4 \mathrm{~B}$ phosphorylation correlates with resistance to PI3K pathway inhibitors, we hypothesized that cell lines with higher levels of activated ERK and/or RSK signaling may maintain higher levels of phosphorylated S6235/236 upon PI3K blockade and thus be relatively insensitive to PI3K inhibition. To investigate this possibility, we surveyed 27 breast cancer cell lines by immunoblotting and queried Oncomine to identify breast cancer cell lines with high levels of RSK4 (Supplemental Figure 4A) (www.oncomine. org). Notably, the 2 breast cancer cell lines exhibiting high levels of 

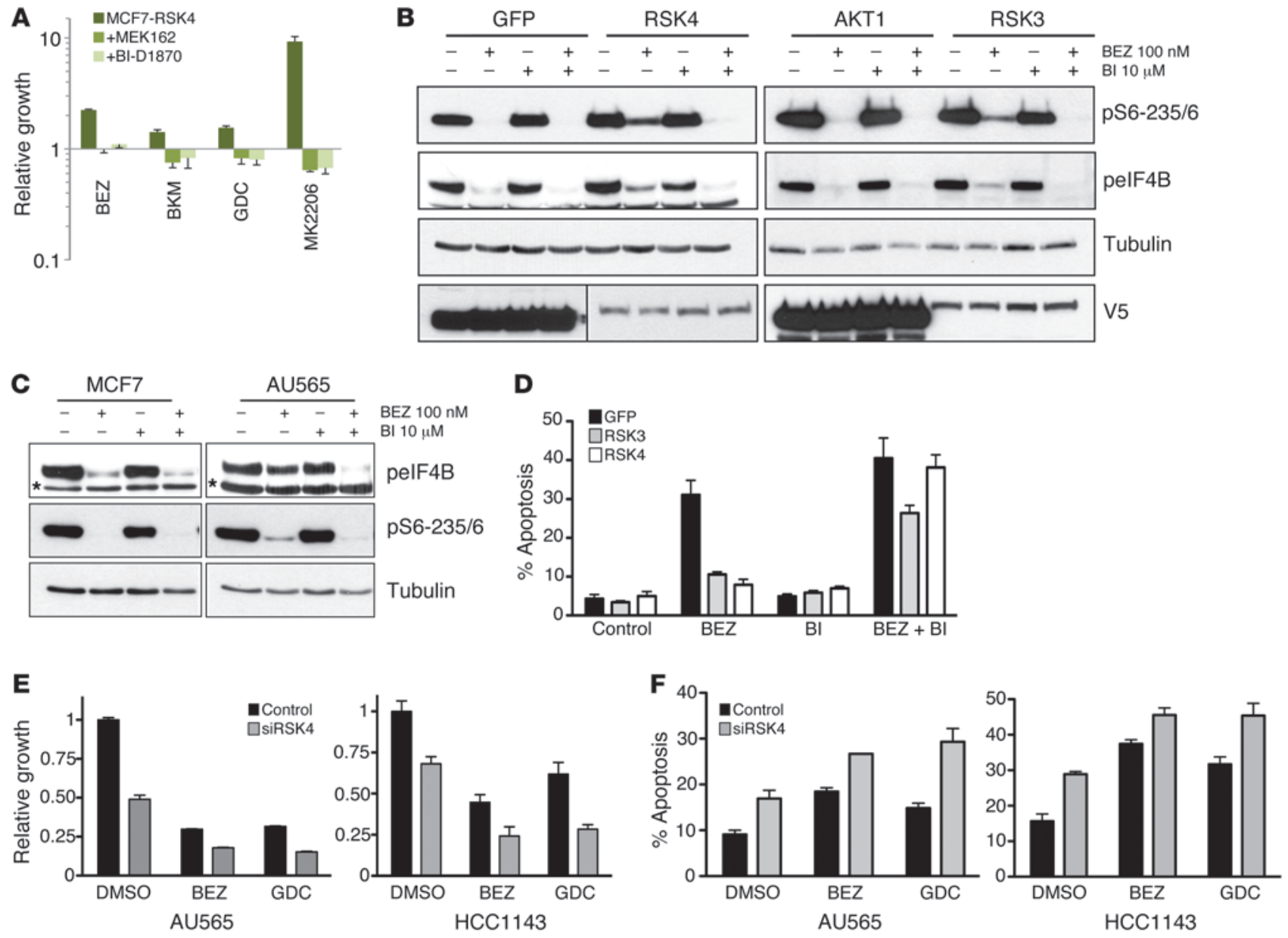

Figure 5

Inhibition of ERK/RSK signaling overcomes resistance to PI3K inhibitors. (A) Quantification of crystal violet staining of RSK4-overexpressing MCF7 cells treated with BEZ235 (200 nM), BKM120 (750 nM), GDC-0941 (1 $\mu \mathrm{M})$, or MK-2206 (2 $\mu \mathrm{M})$ in combination with either MEK162 (1 $\mu$ M) or BI-D1870 $(5 \mu \mathrm{M})$ for 8 days. Bars represent fold increase relative to treated GFP controls (mean \pm SD of 3 independent experiments). (B) MCF7 cells expressing GFP, AKT1, RSK3, or RSK4 were treated with BEZ235 (100 nM) or BI-D1870 $(10 \mu \mathrm{M})$ for 24 hours. V5-tagged proteins were run on the same blot, but bands were noncontiguous due to differences in protein size. (C) MCF7 and AU565 cells were treated with BEZ235 (100 $\mathrm{nM})$ and/or BI-D1870 (10 $\mathrm{MM})$ for 24 hours. Asterisks indicate nonspecific band. (D) MCF7 cells expressing GFP, RSK3, or RSK4 were treated with BEZ235 $(100 \mathrm{nM})$ or BI-D1870 $(10 \mu \mathrm{M})$ for 24 hours and subjected to cell-cycle analysis to assess induction of apoptosis (mean \pm SEM of 3 independent experiments). (E) Proliferation assay of breast cancer cells AU565 and HCC1143 transfected with siRNAs targeting RSK4 or control treated with BEZ235 and GDC-0941 for 24 hours, assessed by CellTiter-Glo (mean \pm SD of 3 independent experiments). (F) AU565 and HCC1143 cells transfected with siRNA targeting RSK4 or control treated with BEZ235 (100 nM) or GDC-0941 (1 $\mu$ M) for 24 hours and subjected to cell-cycle analysis to assess induction of apoptosis (mean \pm SEM of 3 independent experiments).

RSK4 in Oncomine, HCC1143 and HCC38, also demonstrated resistance to the PI3K inhibitor GSK 1059615 (data not shown). As expected, when subjected to treatment with PI3K inhibitors, cell lines with high levels of RSK4 activity exhibited a decrease in sensitivity compared with the sensitive cell line MCF7 (Figure 4C). Furthermore, both AU565 and MDA-MB-231, but not MCF7, retained rpS6 and eIF4B phosphorylation when treated with different PI3K pathway inhibitors (Supplemental Figure 4B). These results suggest that, while rpS6 and eIF4B phosphorylation is principally regulated by the PI3K/AKT/mTOR axis, in the context of RSK overexpression or activation by upstream components, RSKs can sustain rpS6 and eIF4B phosphorylation during PI3K pathway downregulation.

In eukaryotic cells, initiation of protein translation is the major rate-limiting step in protein synthesis (42). Recent studies have suggested that phosphorylation of $\operatorname{Ser}^{235 / 236}$ in $\mathrm{rpS} 6$ and eIF4B $\mathrm{Ser}^{422}$ is required for cap-dependent translation of mRNA (41). To determine the effects of RSK4 overexpression on translation, we monitored new protein synthesis rates in vivo by labeling cells with $\mathrm{S}^{35}$ methionine. Indeed, we observed that RSK4-overexpressing cells had higher levels of total protein synthesis in both normal and PI3K inhibitor-treated conditions compared with control cells (Figure 4, $\mathrm{D}$ and $\mathrm{E})$. Collectively, our data suggest that RSK overexpression prevents response to $\mathrm{PI} 3 \mathrm{~K}$ inhibition through maintenance of protein translation and through the inhibition of apoptosis.

Combination of PI3K and RSK blockade overcomes resistance to PI3K inhibition in RSK-overexpressing cells. The observations described above suggest that activation of the ERK/RSK pathway serves as a mechanism to circumvent PI3K inhibitor sensitivity. Therefore, we 
A

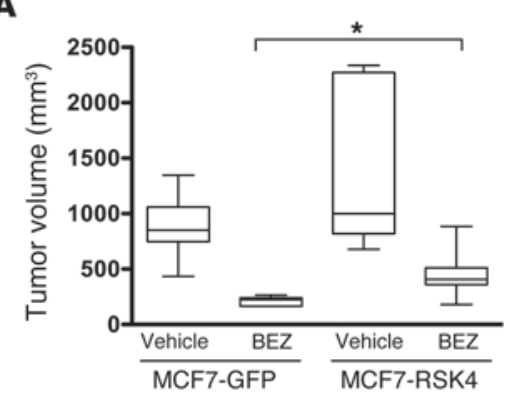

C

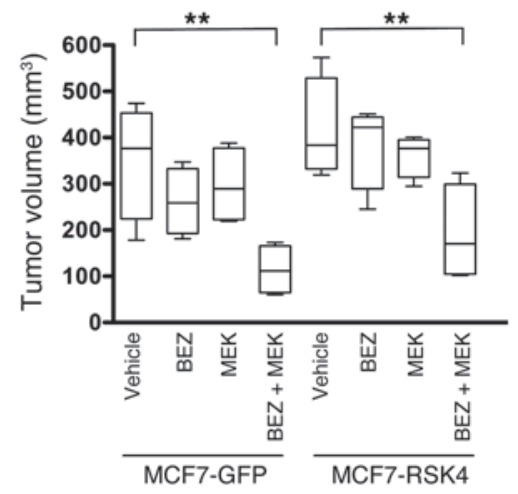

B

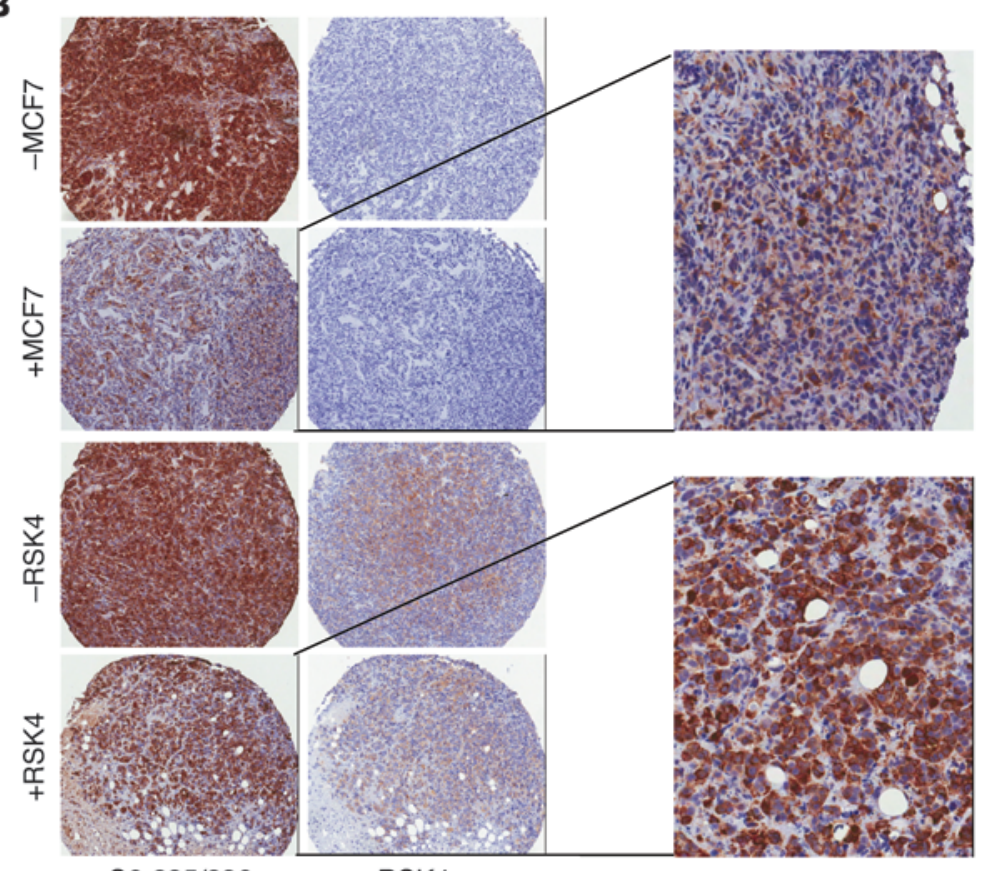

RSK4
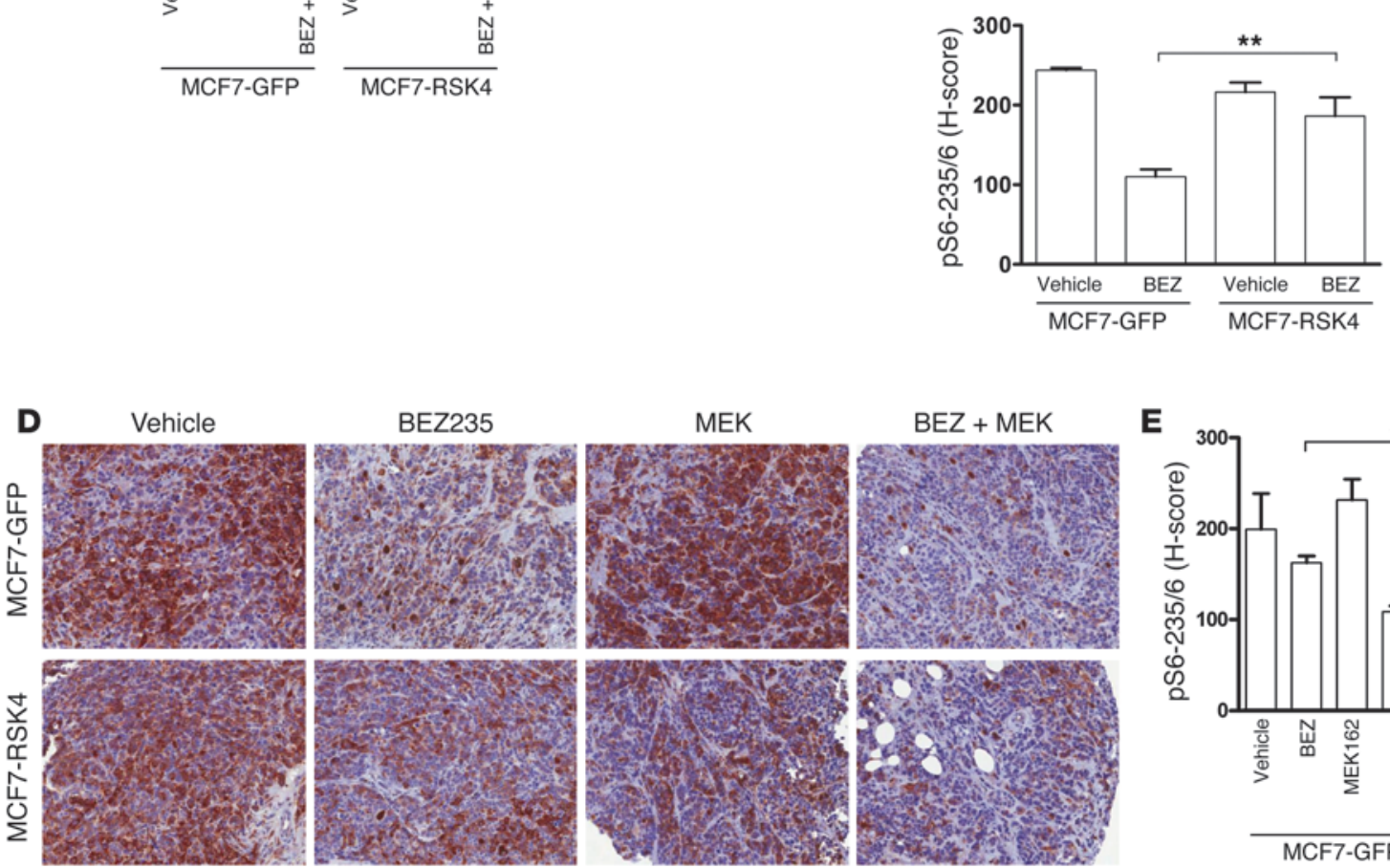

$B E Z+M E K$
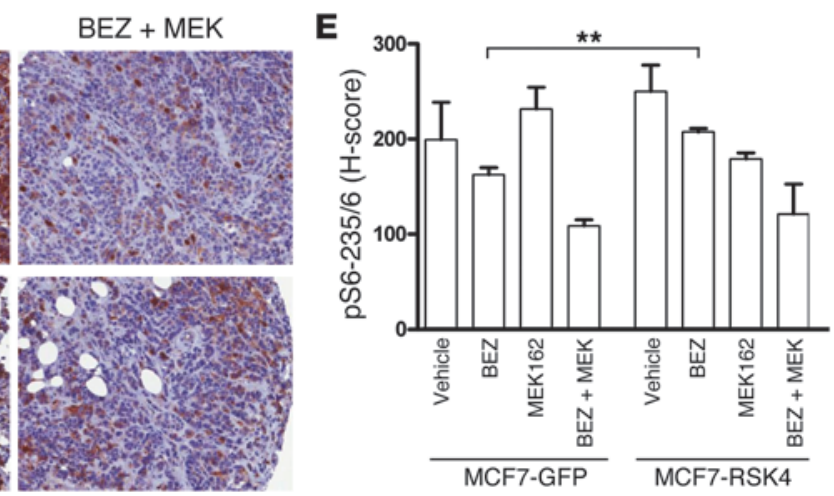

pS6-235/6

\section{Figure 6}

In vivo modeling of RSK4 resistance phenotype and the use of ERK pathway inhibitors to overcome resistance. (A) Mouse xenograft experiment with MCF7 cells overexpressing RSK4 or GFP control. Mice were treated 6 times per week $(6 \mathrm{IW})$ with BEZ235 (30 mg/kg) or vehicle for 24 days (end of experiment). Box plots represent tumor volumes, with whiskers depicting minimum and maximum. A 2-tailed Student's $t$ test compares the 2 treated populations. ${ }^{*} P<0.05$ (mean \pm SEM, $n=8$ ). (B) Tumors were harvested at 24 days and analyzed by IHC for phosphorylation of rpS6 $235 / 236$ and RSK4 expression. Representative images are shown in top panel. H-Score quantification of IHC analysis of rpS6 $6^{235 / 236}$, bottom panel. A 2-tailed Student's $t$ test compares the 2 treated populations. ${ }^{* *} P<0.01$ (mean \pm SEM). Original magnification, $\times 40 ; \times 400$ (enlarged). (C) Mouse xenograft assay with MCF7 cells overexpressing RSK4 or GFP control. Mice were treated 6 times per week with single agent BEZ235 (25 mg/kg, $6 \mathrm{IW})$ or MEK162 (6 mg/kg, twice daily, BID) or in combination. Boxes represent tumor volume variation; lines represent mean tumor volume; bars represent SEM. A 2-tailed Student's $t$ test compares the treated versus untreated tumors. ${ }^{* \star} P<0.01$ (mean $\pm \mathrm{SEM}, n=8$ ). (D) Tumors generated in $\mathbf{C}$ were analyzed by $\mathrm{IHC}$ for phosphorylation of $\mathrm{rpS6} 6^{236 / 236}$. Representative images are displayed. Original magnification, $\times 400$. (E) $\mathrm{H}-\mathrm{Score}$ quantification of IHC analysis of rpS6 $6^{235 / 236}$ of tumors from Figure 5C, A 2-tailed Student's $t$ test compares the 2 treated populations. ${ }^{* \star} P<0.01$ (mean \pm SEM). 
hypothesized that the dual blockade of PI3K and RSK pathways would reverse the resistance phenotype and the molecular markers associated with resistance seen in RSK-overexpressing cells. To test this hypothesis, we combined PI3K inhibitors with the MEK inhibitor NVP-MEK162 (MEK-162) (43) or the pan-RSK-specific inhibitor dihydropteridinone (BI-D1870) (44). In MCF7 cells, RSK3 or RSK4 expression decreased response to treatment with any of the PI3K inhibitors alone. However, the combination of PI3K inhibition with MEK162 or BI-D1870 completely reversed the resistance of RSK-expressing cells (Figure 5A and Supplemental Figure 5A). BI-D1870 has previously been demonstrated to inhibit the cellcycle regulators PLK1 and Aurora B, albeit at much higher concentrations than RSK inhibition (45). To verify the specific efficacy of BI-D1870, we treated AKT-overexpressing cells with combined PI3K inhibitors and RSK or MEK inhibitors. As expected, MCF7 cells overexpressing AKT1 were refractory to combined PI3K and MEK/RSK inhibition, confirming the specific efficacy of this combination for cells with activation of the MEK/ERK/RSK pathway (Supplemental Figure 5B). We observed that rpS6 and eIF4B phosphorylation was completely attenuated only when MCF7-RSK cells were treated with the combination of BEZ235 and BI-D1870 or another MEK inhibitor (AZD6244) (46), in agreement with the effects on cell viability (Figure 5B and Supplemental Figure 6A). Accordingly, we also observed an inhibition of RSK phosphorylation at $\mathrm{Ser}^{380}$, which serves as a marker of RSK activity, in MCF7RSK4 cells upon treatment with AZD6244 or MEK162, verifying that MEK inhibition downregulates the function of overexpressed RSK (Supplemental Figure 6A). Furthermore, combined inhibition of PI3K and RSK diminished rpS6 phosphorylation levels and proliferation compared with either inhibitor alone in breast cancer cell lines with high levels of RSK (Figure 5C and Supplemental Figure 6B). Since RSK4 overexpression renders cells resistant to the proapoptotic effects of PI3K inhibitors, we hypothesized that combined inhibition of RSK and PI3K would enhance apoptosis compared with either compound alone. Indeed, combined inhibition of PI3K and RSK significantly enhanced apoptosis to levels similar to those in control GFP-overexpressing cells compared with RSK4-overexpressing MCF7 cells and in breast cancer cell lines exhibiting elevated levels of RSK4 (Figure 5D and Supplemental Figure 6C). Similarly, targeted knockdown of RSK4 increased the sensitivity to PI3K inhibition in multiple RSK4-overexpressing breast cancer cell lines, substantiating the role of RSK4 in mediating resistance to PI3K inhibition (Figure 5, E and F, and Supplemental Figure 6, D and E). Importantly, the degree of apoptosis was virtually identical in RSK4 knockdown cells versus MEK inhibition when combined with a PI3K inhibitor (Supplemental Figure 7, A and B). Furthermore, combined inhibition of PI3K with either BI-D1870 or MEK inhibition inhibited protein translation specifically in RSK-expressing cells and restored inhibition of protein translation upon PI3K inhibition (Supplemental Figure 7, C and D). Collectively, our data suggest that the combination of PI3K and RSK pathway inhibitors is effective at decreasing rpS6 and eIF4B phosphorylation, overall translation, and survival in cells with altered RSK activity.

RSK expression promotes resistance to PI3K inhibitors in vivo. Next, we sought to analyze the tumorigenic potential of RSK4-overexpressing cells and response to BEZ235 in a xenograft model. To this end, we injected immunodeficient mice with MCF7 cells overexpressing RSK4 or GFP as a control. BEZ235 treatment at $30 \mathrm{mg} / \mathrm{kg}$ was started 7 days after injection, when tumors reached an average volume of $250 \mathrm{~mm}^{3}$. RSK4-overexpressing cells exhibited growth rates similar to those of control cells in vehicle-treated mice (Figure 6A). In contrast, and in consonance with previous results in vitro, RSK4 overexpression allowed tumors to progress even in the presence of BEZ235 (Figure 6A). Furthermore, RSK4 expression led to robust retention of $\mathrm{rpS} 6$ phosphorylation in tumors in the presence of BEZ235, as measured by phospho-rpS6 staining (Figure 6B). To determine whether the resistance phenotype of RSK-overexpressing tumors extends to other PI3K pathway inhibitors, we further determined the sensitivity of these tumors to BKM120 and MK-2206. As observed in vitro, treatment with all PI3K pathway inhibitors completely blocked the proliferation potential of control tumors. However, RSK4-overexpressing tumors decreased the growth inhibitory properties of all the PI3K inhibitors tested (Supplemental Figure 8A). Since RSK4 expression diminished the effectiveness of single-agent PI3K treatment, we explored the antitumor activity of PI3K inhibition in combination with ERK/RSK pathway inhibitors. We analyzed tumor growth inhibition of MCF7-RSK4-derived xenografts in response to the combination of BEZ235 and the MEK inhibitor MEK162. As the BEZ235 concentration had to be reduced in these experiments from $30 \mathrm{mg} / \mathrm{kg}$ to $25 \mathrm{mg} / \mathrm{kg}$ to compensate for general toxicity of the combination treatments, the difference in drug response between RSK4- and GFP-expressing animals was less pronounced than in the single-agent experiments. Nevertheless, RSK4-overexpressing cells exhibited a clear trend toward decreased responsiveness to BEZ235 as single-agent therapy compared with the control cells (Figure 6C). When MEK162 was combined with BEZ235, a significant reduction of tumor growth was observed $(P=0.01)$. This increase in antitumor activity was accompanied by a decrease in phospho-ERK and phospho-S6 staining (Figure 6, D and E, and Supplemental Figure 8B). No significant changes were observed in phospho-4EBP1 staining, a direct target of mTOR activity (Supplemental Figure 8, C and D).

Because the intrinsic properties of artificially cultured cell lines tend to diverge from the characteristics of real tumors, we confirmed our results in PDXs. These PDXs generate tumors with the same histopathological characteristics and oncogenic mutations as found in the human patient from whom they were derived (data not shown) (47). Protein lysates of 11 triple-negative PDXs were assessed for pRSK-380 by immunoblotting (data not shown). Of the 11 models, we identified the 2 PDXs that exhibited the greatest difference in levels of activated RSK, PDX60 (high RSK) and PDX156 (low RSK) (Figure 7A).

In concordance with our previous data, the PDX that exhibited hyperactivation of RSK4 remained relatively insensitive to inhibition with the PI3K inhibitor BKM120, while the PDX with low levels of RSK activity were acutely sensitive to PI3K inhibition (Figure 7B). Western blot and reverse phase protein analysis (RPPA) of these PDXs confirmed that following PI3K inhibitor treatment, PDX156 tumors had reduced phospho-S6235/236 levels whereas PDX60 tumors maintained high levels of phospho-S6 $6^{235 / 236}$ (Figure 7C and Supplemental Figure 9A). Furthermore, combined inhibition of PI3K and MEK in PDX60 significantly decreased phospho-S6 $6^{235 / 236}$ and overall tumor volume compared with either inhibitor alone (Figure 7, D and E). Taken together, our data suggest that hyperactivation of RSK may limit PI3K inhibitor function in breast cancer patients.

To further assess the potential clinical relevance of RSK function in breast cancer, we investigated RSK activity, as assessed 

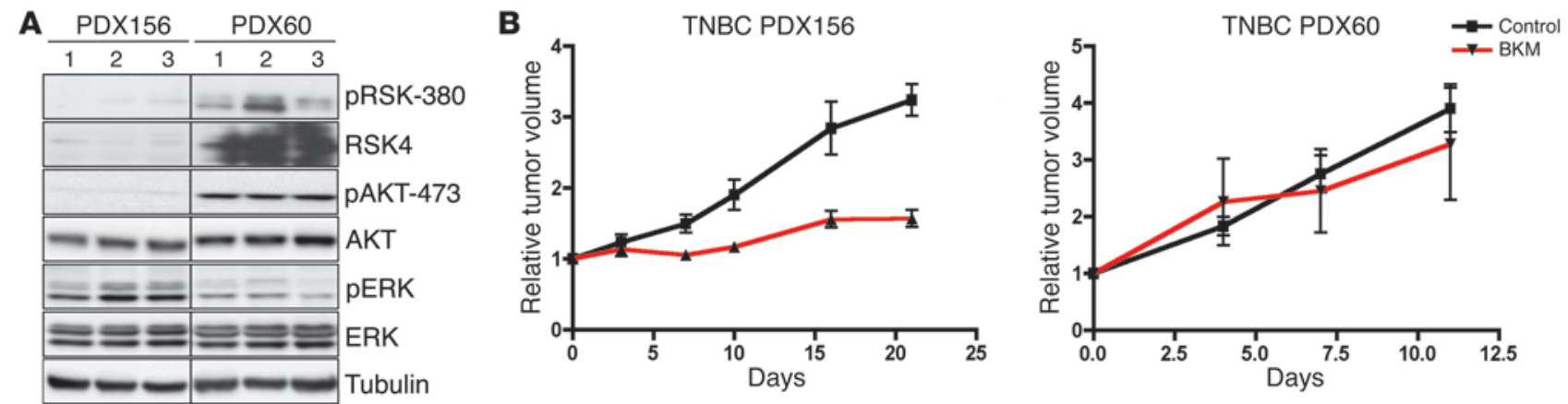

C

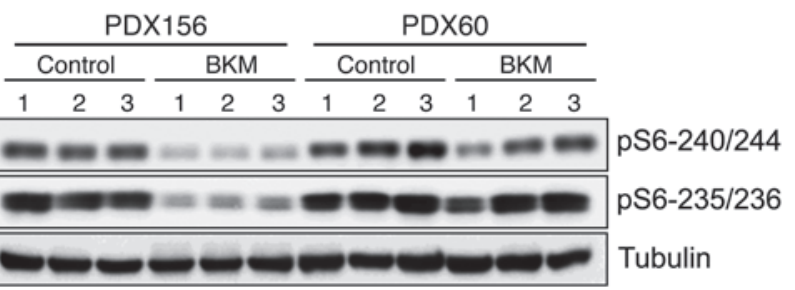
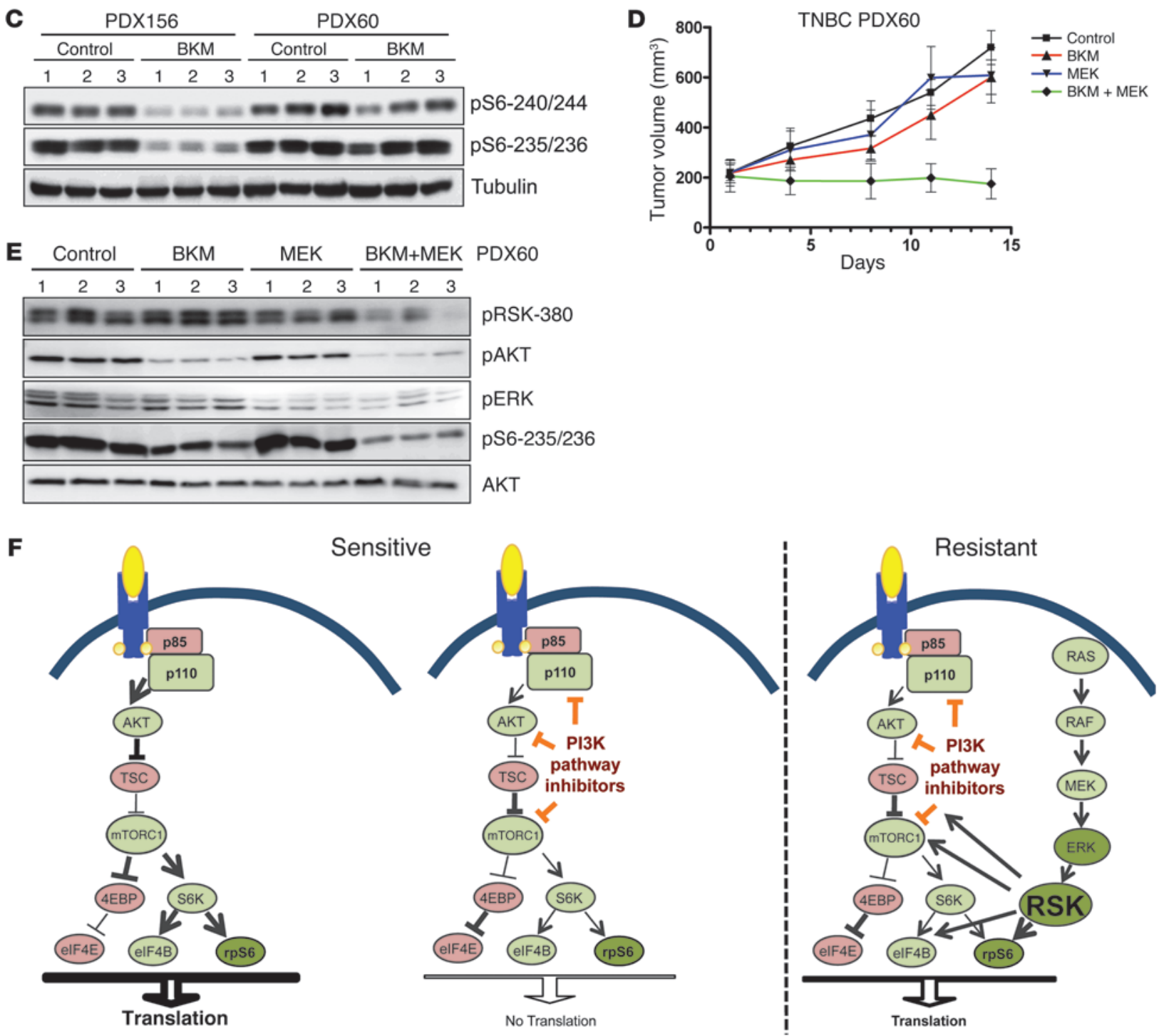

Figure 7

In vivo modeling of RSK4 resistance in PDXs. (A) Western blot analyses of PDX60 (high RSK) and PDX156 (low RSK). Tumor-derived extracts from 3 individual tumors were analyzed with the indicated antibodies. (B) Patient-derived xenograft assay with PDX156 (low RSK, left panel) and PDX 60 (high RSK, right panel). Mice were treated daily with BKM120 (27.5 mg/kg) or vehicle (mean \pm SEM, $n=8)$. (C) Western blot analysis of PDX156 and PDX60 tumors treated with DMSO or BKM120. Tumor-derived extracts from 3 individual tumors were analyzed with the indicated antibodies. (D) Patient-derived xenograft assay with PDX60 tumor treated with DMSO, BKM120, MEK, or a combination (mean \pm SEM, $n=8$ ). (E) Western blot analysis of PDX tumors treated with DMSO, BKM120, MEK162, or a combination. Tumor-derived extracts were analyzed with the indicated antibodies. (F) Schematic overview of PI3K/mTOR and ERK/RSK pathways converging to regulate S6 phosphorylation and translation. Observations presented here support a model in which aberrant activation of the ERK/RSK signaling axis contributes to S6 phosphorylation, translation initiation, and resistance to PI3K/mTOR blockade. 
by phosphorylation of $\mathrm{Thr}^{359} / \mathrm{Ser}^{363}$, across a panel of breast invasive tumors from the TCGA tumor bank (http://tcga-data. nci.nih.gov/tcga/) for which RPPA data was available (48). We observed elevated levels of phospho-RSK in a subset of basal-like, HER2-enriched, luminal A, and luminal B breast tumors, suggesting RSK is hyperactivated in at least some tumors of these subtypes (Supplemental Figure 9B). Moreover, basal-like tumors as a group had significantly higher levels of phospho-RSK compared with the rest of tumor samples, in agreement with the observation that basal-like breast tumors exhibit evidence of RAS/MEK/ ERK pathway activation (Supplemental Figure 9B and refs. 10, 49). We also interrogated the Human Protein Atlas (http://www. proteinatlas.org) for expression levels of RSK3 and RSK4 based on immunohistochemical (IHC) staining of tumor samples (50). Here, we observed frequent strong staining for RSK4, and to a lesser degree RSK3, across a number of tumor types, including breast, colorectal, prostate, thyroid, urothelial, and lung cancers (RSK3: http://www.proteinatlas.org/ENSG00000071242; RSK4: http://www.proteinatlas.org/ENSG00000072133). Finally, we determined the frequency of amplification or overexpression of RSK3 and RSK4 in a panel of breast cancer cell lines, using the Broad-Novartis Cancer Cell Line Encyclopedia (CCLE) (http:// www.broadinstitute.org/ccle/home) (51). We queried 59 breast cancer cell lines and observed that RSK3 and RSK 4 transcripts are upregulated in $8 \%$ and $46 \%$ of breast cancer cell lines, respectively (Supplemental Figure 9C). Taken together, these observations suggest that RSK3 and RSK4 may be functionally important in breast tumorigenesis.

\section{Discussion}

Inhibitors targeting the PI3K pathway have the potential to be effective anticancer agents and, as such, are being developed at a rapid pace. However, previous experience with targeted therapies predicts that patients who initially respond invariably relapse due to acquisition of drug resistance. To anticipate mechanisms of resistance to PI3K inhibitors, we have screened a library of kinase ORFs and have identified a number of kinases that circumvent PI3K inhibitor sensitivity. Validated candidates included potent activators of PI3K and ERK signaling pathways, such as ERBB2 and IGF1R, as well as downstream effectors AKT1 and AKT3. In addition, we have identified the RSK family members RSK3 and RSK4 as repressors of PI3K inhibitor function. Functional studies have implicated RSKs in the regulation of diverse cellular processes, including transcription, translation, survival, cell-cycle progression, and migration, through phosphorylation of targets including CREB, GSK3, TSC2, rpS6, raptor, eIF4B, BAD, and p27, among others $(26,41,52-54)$. The RSKs have all been linked with tumorigenesis, albeit in different contexts. RSK1 and RSK2 have been reported as overexpressed in breast and prostate cancer, while RSK3 has been proposed to be a tumor suppressor in ovarian cancer (55-57). RSK4 has previously been characterized as essential for $\mathrm{p} 53$-dependent proliferation arrest as well as stress- and oncogene-induced senescence $(28,58,59)$. Interestingly, the RSK4 isoform exhibits constitutively high activity, is upregulated in MMTV-Myc mouse breast tumors, is aberrantly expressed in breast cancer, and has been implicated in sunitinib resistance $(58,60-63)$. Here, we demonstrate that RSK3 and RSK4 can also mediate resistance to PI3K inhibitors in breast cancer cells both in vitro and in vivo.

Our observations strongly support a role for retention of rpS6 and eIF4B phosphorylation in the resistance phenotype of
RSK-overexpressing cells, in agreement with a previous report noting retention of $\mathrm{rpS} 6$ phosphorylation in breast cancer cell lines exhibiting intrinsic resistance to PI3K inhibition (9). Previous studies have suggested that RSKs directly phosphorylate rpS6 at $\mathrm{Ser}^{235 / 236}$ and eIF4B at $\mathrm{Ser}^{422}$. The former promotes binding of $\mathrm{rpS} 6$ to the 7-methylguanosine cap complex and enables cap-dependent translation to proceed, while the latter is critical for eIF4B binding to the cap complex and enhanced helicase activity of eIF4A and increased cellular translation (64-66). In agreement with these results, we observed that RSK4-overexpressing cells exhibited elevated levels of overall translation, which are maintained in the presence of PI3K inhibitors (Figure 7F). These results are also consistent with a previous report implicating upregulation of cap-dependent translation by eIF4E amplification in promoting resistance to BEZ235 (67).

As RSKs are directly regulated by RAF/MEK/ERK signaling, we hypothesized that inhibition of this pathway would overcome the resistance phenotype of RSK-overexpressing cells and reverse all associated cellular phenotypes. We observed that addition of MEK or RSK inhibitors restored responsiveness of RSK-expressing cells to PI3K inhibitors by all parameters analyzed, including translation, 66 phosphorylation, cell viability, and in vivo tumor formation (Figures 5-7 and Supplemental Figures 5-8). Importantly, this reversal of phenotype was specific for RSKs, as AKT1-overexpressing cells remained refractory to PI3K inhibition even with the addition of MEK or RSK inhibitors.

One potential limitation of this study is the fact that we were unable to examine RSK inhibition, either through chemical inhibition or knockdown of RSK4, in relevant xenograft models. This is primarily due to the technical difficulty of the experiments and the lack of suitable chemical reagents currently available. Significantly, however, in both in vitro and in vivo experiments, MEK inhibitors inhibited RSK phosphorylation (Figure 7E, Supplemental Figure 6A, and data not shown), indicating that the MEK inhibitors used in our animal models effectively inhibited RSK activity. Collectively, our data suggest that RSK overexpression renders tumors insensitive to PI3K inhibition, which can be overcome by inhibiting the MEK/ERK/RSK pathway.

The observations presented here support the notion that breast cancer cells upregulate overall protein translation and cell proliferation through overlapping but parallel pathways, the PI3K/mTOR and ERK/RSK pathways (Figure 7F). Interestingly, another significant outlier in our screen, the protooncogene PIM2, regulates key effectors of cap-dependent translation, including eIF4E, 4EBP1, and $\mathrm{S} 6 \mathrm{~K}$, independently of the $\mathrm{PI} 3 \mathrm{~K} / \mathrm{mTOR}$ pathway, supporting the notion that combined pharmacological inhibition of multiple translational regulators should be explored (Figure 7F) (68).

A number of reports have recently shown that an elevated ERK activation signal, either through intrinsic KRAS mutations or through the activation of compensatory feedback loops observed following PI3K inhibition, limits the effectiveness of PI3K inhibitors in the clinic (13-20). Early clinical trials assessing the effectiveness of PI3K and MEK inhibitors have demonstrated some evidence of efficacy in certain tumor types (69). However, initial reports seem to suggest that the use of MEK inhibitors in the clinic results in undesired toxicities, limiting the effectiveness of this compound (70). Importantly, our studies suggest that targeted RSK inhibition is as effective as MEK inhibition when used in combination with PI3K inhibitors, resulting in similar degrees of decreased proliferation and augmented apoptosis. As RSK-specific 
inhibitors target only a single effector arm of MAPK signaling, they may provide a therapeutic window circumventing many of the potential toxicities associated with current MEK-PI3K inhibitor combination strategies. Moreover, we anticipate that use of this combination will also be indicated in the treatment of tumors that exhibit evidence of MEK/ERK-driven signaling.

\section{Methods}

Kinase ORF screen. Kinase library ORFs and GFP controls were expressed from pLX-Blast-V5 lentiviral expression vectors, which confer blasticidin resistance, as previously described (34). Virus was produced by transfecting $239 \mathrm{~T}$ cells in 96-well plates, and screening infections were performed in 384-well plates in octuplicate, using standard spin-infection protocols with 1 ORF per well, as previously described (Figure 1A and ref. 34). Medium was changed 24 hours after infection to $10 \mu \mathrm{g} / \mathrm{ml}$ blasticidin, 200 nM BEZ235, $1 \mu \mathrm{M}$ BKM120, or no drug, with 2 replicates per condition (Figure 1A). Five days after medium change, cell viability was assessed with CellTiter-Glo (Promega). Duplicates were averaged for all subsequent analysis. Infection efficiency was monitored by comparing plates selected with blasticidin with untreated plates, and those wells with greater than 2 -fold difference in cell number between the 2 conditions were eliminated from the analysis. By this criterion, approximately $95 \%$ of the ORF library was efficiently transduced into the target cells and thus tested for phenotype (Supplemental Figure 1A).

Cell culture. MCF7 and MDA-MB-231 cells were maintained in DMEM (Gibco; Invitrogen) supplemented with $10 \% \mathrm{FBS}$ at $37^{\circ} \mathrm{C}$ in $5 \% \mathrm{CO}_{2}$. BT474 and AU565 cells were maintained in RPMI medium supplemented with $10 \% \mathrm{FBS}$ at $37^{\circ} \mathrm{C}$ in $5 \% \mathrm{CO}_{2}$. All cells were obtained from ATCC. Stable cell lines were maintained in appropriate medium supplemented with $10 \mu \mathrm{g} / \mathrm{ml}$ blasticidin.

Cell viability and sub-G $G_{1}$ assays. MCF7 cells infected as indicated were seeded in 12-well plates $\left(2 \times 10^{4}\right)$. After 24 hours, cells were treated with BEZ235 (100 or $200 \mathrm{nM})$, BKM120 (0.75 or $1 \mu \mathrm{M})$, GDC-0941 (1 $\mu \mathrm{M})$, or MK2206 $(2 \mu \mathrm{M})$ alone or in combination with MEK162 $(1 \mu \mathrm{M})$, BI-D1870 (10 $\mu \mathrm{M})$, or AZD6244 $(1 \mu \mathrm{M})$, as indicated in text. Cell numbers were quantified by fixing cells with $4 \%$ glutaraldehyde or methanol, washing the cells twice in $\mathrm{H}_{2} \mathrm{O}$, and staining the cells with $0.1 \%$ crystal violet (Sigma-Aldrich). The dye was subsequently extracted with $10 \%$ acetic acid, and its absorbance was determined $(570 \mathrm{~nm})$. Growth curves were performed in triplicate. Viability assays with CellTiter-Glo (Promega) were performed by plating 2,000 cells in 96-well plates, adding the drug at 24 hours, and assaying 4 to 5 days after drug addition. Cell-cycle and hypodiploid apoptotic cells were quantified by flow cytometry as described (71). Briefly, cells were washed with PBS, fixed in cold $70 \%$ ethanol, and then stained with propidium iodide while being treated with RNase (Sigma-Aldrich). Quantitative analysis of sub- $\mathrm{G}_{1}$ cells was carried out in a FACScalibur cytometer using Cell Quest software (BD Biosciences).

Western blotting and quantification. Cells were lysed in solubilizing buffer (50 mM Tris, pH 8.0, $150 \mathrm{mM} \mathrm{NaCl}, 1$ \% NP-40, 0.5\% deoxycholic acid, $0.1 \%$ SDS, $1 \mathrm{mM}$ sodium vanadate, $1 \mathrm{mM}$ pyrophosphate, $50 \mathrm{mM}$ sodium fluoride, $100 \mathrm{mM} \beta$-glycerol phosphate) supplemented with protease inhibitors (Roche). Whole-cell extracts were then separated on SDS-PAGE gels and transferred to polyvinylidene difluoride membranes (Millipore). Membranes were blocked with bovine serum albumin and probed with specific antibodies. Blots were then incubated with an HRP-linked second antibody and resolved with chemiluminescence (Pierce). Western blots were quantified using ImageJ (http://rsb.info.nih.gov/ij/).

Plasmids and reagents. Antibodies against PARP, cleaved PARP, cleaved caspase 7, phospho-AKT, AKT, phospho-ERK, ERK, phospho-S6235/236, phospho-S6240/244, phospho-eIF4B-422, phospho-GSK3, phospho-p70
S6K-389, phospho-4EBP1-37/46, and phospho-RSK-380 were from Cell Signaling Technology. Antibodies against cyclin D1, GapdH, and tubulin were from Santa Cruz Biotechnology Inc. Antibodies against total V5 were from Invitrogen. BEZ235, BKM120, and MEK162 were provided by Emmanuelle Di Tomaso and Michel Maira (Novartis Institutes for Biomedical Research). GDC-0941, MK-2206, and AZD6244 were purchased from Selleck. BI-D1870 was purchased from Axon Medcam. Cycloheximide was purchased from Sigma-Aldrich. siRNA-targeting RSK4 was purchased from Dharmacon and transfected according to the manufacturer's protocols.

Metabolic labeling and quantification. MCF7 cells were grown to $70 \%$ confluence in 10-cm plates and either incubated overnight in $10 \%$ serum or exposed to BEZ235 (200 nM), BKM120 (750 nM), GDC0941 (1 $\mu \mathrm{M})$, or cycloheximide in $10 \%$ serum. Cells were then washed once with DMEM lacking cysteine and methionine. DMEM lacking cysteine and methionine but including dialyzed serum and kinase inhibitors as indicated was added. Cells were incubated for 1 hour, 250 pCi of Expre35S35S (NEN) was added to each well, and the cells were labeled for a further 30 minutes. Cells were washed once with ice-cold PBS, and whole-cell extracts were isolated as described above and separated by SDS-PAGE. The 35S-labeled proteins were visualized by autoradiography with film. The amount of $35 \mathrm{~S}$ incorporated into protein was measured using a Beckman LS6500 Scintillation Counter.

Tumor xenografts. Six-week-old female athymic nude Foxn $1^{\text {nu }}$ mice were purchased from Harlan Laboratories. Mice were housed in air-filtered laminar flow cabinets with a 12-hour light/12-hour dark cycle and given food and water ad libitum. Mice were handled with aseptic procedures and allowed to acclimatize to local conditions for 1 week before the experimental manipulations. A $17 \beta$-estradiol pellet (Innovative Research of America) was implanted subcutaneously into each mouse 1 day before cell injection. $10^{7}$ MCF-GFP or MCF7-RSK4 cells were resuspended in PBS/Matrigel (BD Biosciences) (1:1) and injected subcutaneously into the right flank of each mouse in $200 \mu \mathrm{l}$ of final volume. Treatments began when tumors reached an average size of $250 \mathrm{~mm}^{3}$ and were thus considered as established growing xenografts. Mice were treated once daily with placebo, BEZ235, BKM120, MK-2206, or MEK162 by oral gavage. BEZ235 (25-30 mg/kg, 6IW [ 6 days on 1 day off]) and BKM120 (30 mg/kg, 6IW) were dissolved in 10\% NMP-90\% PEG, freshly formulated, and administrated within 30 minutes. MK-2206 (100 mg/kg, 3IW) was formulated in 30\% Captisol and MEK162 (6 mg/kg, BID) in 0.5\% Tween-80, 1\% carboxymethyl cellulose. For tumor growth studies, mice were treated for 7-24 days, depending on the xenograft model and treatment regime. Tumor xenografts were measured with calipers 3 times a week, and tumor volume was determined using the following formula: (length $\times$ width $\left.{ }^{2}\right) \times(\pi / 6)$. At the end of the experiment, the animals were anesthetized with $1.5 \%$ isofluorane-air mixture and killed by cervical dislocation. Tumors were removed 2 hours following the last administration.

IHC. Tumor xenografts or human breast cancer tumors were fixed immediately after removal in a $10 \%$ buffered formalin solution for a maximum of 24 hours at room temperature before being dehydrated and paraffin embedded under vacuum conditions. Tissue microarrays (TMA) were constructed, including triplicated cores from each xenograft. TMA slides underwent deparaffinization and antigen retrieval using the PTLink system (DAKO) following the manufacturer's instructions. Primary antibodies were phospho-rpS6-Ser ${ }^{235 / 236}$, phospho-ERK-Thr ${ }^{202} / \mathrm{Tyr}^{204}$, phopsho-4EBP1 37/46 (all from Cell Signaling Technology), or RSK4 (Millipore). Samples were incubated with a 1:40 solution of streptavidin/ peroxidase for 30 minutes. Staining was developed with freshly prepared $0.05 \%$ 30,3-diaminobenzidine tetrahydrochloride, which was then counterstained with hematoxylin. No labeling was observed in control experiments when primary antibodies were omitted or, alternatively, when normal nonimmune serum was used. There was no evidence of cross-reactivity 
with the antibodies used in this study. For IHC evaluation, H-scores were used to quantify the expression of the phosphoproteins.

Establishment and selection of PDXs in nude mice. Following implantation and engraftment of a metastatic patient needle biopsy $(3 \times 3 \mathrm{~mm})$ in 6-week-old female HsdCpb:NMRI-Foxn1nu mice (Harlan Laboratories), a PDX colony was established and checked for expression of immunohistopathological markers in agreement with the original biopsy. Animals were supplemented with $1 \mu \mathrm{M}$ estradiol (Sigma-Aldrich) in the drinking water. Protein lysates of 11 triple-negative PDXs were assessed for pRSK-380 by Western immunoblot (not shown). Of the 11 models, the PDXs expressing the highest (PDX60) or lowest (PDX156) levels of PRSK-380 were selected for in vivo testing of BKM120 antitumor response. Briefly, following an expansion phase by sequential implantation, tumors were implanted into nude mice for experimentation. Once tumors reached a mean size of $250 \mathrm{~mm}^{3}$, mice were distributed in groups of similar mean and SEM and treated by oral gavage with BKM120 6xQD (dissolved in NMP-PEG) or AZD6244 (dissolved in Methylcellulose/Tween). Tumor grafts were measured with calipers, and tumor volumes were determined using the following formula: (length $\times$ width $\left.^{2}\right) \times(\pi / / 6)$. At the end of the experiment, animals were sacrificed by $\mathrm{CO}_{2}$ inhalation. Tumor volumes are plotted as relative to day 1 and expressed as mean \pm SEM of the group.

Statistics. All statistics were calculated using GraphPad Prism or Microsoft Excel. The tests used include 2-tailed $t$ test, SD, SEM, 1-way ANOVA, and log-rank test where appropriately indicated in figure legends. $P \leq 0.05$ was considered statistically significant. All $P$ values are depicted in the figures or in the figure legends.

Study approval. Patient consent for tumor use in animals was obtained under a protocol approved by the Vall d'Hebron Hospital Clinical Investigation Committee and Animal Use Committee. Mice were maintained and treated in accordance with protocols approved by the Vall d'Hebron University Hospital Care and Use Committee.

\section{Acknowledgments}

The authors thank Anna Schinzel of the DFCI RNAi facility for assistance with reagents and Sadhna Vora and Jeff Engelman for helpful discussion. The authors also thank Emmanuelle Di Tomaso and Michel Maira (Novartis Institutes for Biomedical Research, Cambridge, Massachusetts, USA, and Basel, Switzerland) for the supply of BEZ235 and BKM120. S.Y. Kim thanks Joseph Nevins for advice and generous support. This research was supported by the Breast Cancer Research Foundation (to J. Baselga), the European Research Council (AdG09250244 to J. Baselga), the Instituto de Salud Carlos III (Intrasalud PSO9/00623 to J. Baselga), a noncommercial research agreement with Novartis (to J. Baselga), FERO foundation supporting research grants (to J. Baselga), the DFCI-Novartis Drug Discovery Program (to W.C. Hahn), R01 CA130988 (to W.C. Hahn), RC2 CA148268 (to W.C. Hahn), U54CA112962 (to W.C. Hahn), and the Snyder Medical Research Foundation (to W.C. Hahn).

Received for publication August 15, 2012, and accepted in revised form February 27, 2013.

Address correspondence to: So Young Kim, Duke University Medical Center, Box 3053, Durham, North Carolina 27710, USA. Phone: 919.684.7955; Fax: 919.613.8646; E-mail: soyoung.kim@duke.edu. Or to: José Baselga, Memorial Sloan-Kettering Cancer Center, 1275 York Avenue, Box 20, New York, New York 10065, USA. Phone: 646.888.4599; Fax: 646.422.0586; E-mail: baselgaj@mskcc.org.
1. Engelman JA. Targeting PI3K signalling in cancer: opportunities, challenges and limitations. Nat Rev Cancer. 2009;9(8):550-562.

2. Liu P, Cheng H, Roberts TM, Zhao JJ. Targeting the phosphoinositide 3-kinase pathway in cancer. Nat Rev Drug Discov. 2009;8(8):627-644.

3. Wong KK, Engelman JA, Cantley LC. Targeting the PI3K signaling pathway in cancer. Curr Opin Genet Dev. 2009;20(1):87-90.

4. Garcia JM, et al. Promoter methylation of the PTEN gene is a common molecular change in breast cancer. Genes Chromosomes Cancer. 2004;41(2):117-124

5. Goel A, et al. Frequent inactivation of PTEN by promoter hypermethylation in microsatellite instability-high sporadic colorectal cancers. Cancer Res. 2004;64(9):3014-3021.

6. Motzer RJ, et al. Efficacy of everolimus in advanced renal cell carcinoma: a double-blind, randomised, placebo-controlled phase III trial. Lancet. 2008; 372(9637):449-456.

7. Yao JC, et al. Everolimus for advanced pancreatic neuroendocrine tumors. N Engl J Med. 2011; 364(6):514-523.

8. Baselga J, Campone M, Piccart M, Burris HA. Everolimus in postmenopausal hormone-receptor-positive advanced breast cancer. $N$ Engl J Med. 2012; 366(6):520-529.

9. Brachmann SM, et al. Specific apoptosis induction by the dual PI3K/mTor inhibitor NVP-BEZ235 in HER2 amplified and PIK3CA mutant breast cancer cells. Proc Natl Acad Sci US A. 2009;106(52):22299-22304.

10. O'Brien C, et al. Predictive biomarkers of sensitivity to the phosphatidylinositol 3 ' kinase inhibitor GDC-0941 in breast cancer preclinical models. Clin Cancer Res. 2010;16(14):3670-3683.

11. Serra V, et al. NVP-BEZ235, a dual PI3K/mTOR inhibitor, prevents PI3K signaling and inhibits the growth of cancer cells with activating PI3K mutations. Cancer Res. 2008;68(19):8022-8030.
12. Juric D, et al. BYL719, a next generation PI3K alpha specific inhibitor: Preliminary safety, PK, and efficacy results from the first-in-human study. Cancer Res. 2012;72(8 supplement):CT-01.

13. Engelman JA, et al. Effective use of PI3K and MEK inhibitors to treat mutant Kras G12D and PIK3CA H1047R murine lung cancers. Nat Med. 2008; 14(12):1351-1356.

14. Janku F, et al. PI3K/AKT/mTOR inhibitors in patients with breast and gynecologic malignancies harboring PIK3CA mutations. J Clin Oncol. 2012; 30(8):777-782.

15. Garrido-Laguna I, et al. KRASness and PIK3CAness in patients with advanced colorectal cancer: outcome after treatment with early-phase trials with targeted pathway inhibitors. PLoS One. 2012; 7(5):e38033.

16. Janku F, et al. PIK3CA mutations in patients with advanced cancers treated with $\mathrm{PI} 3 \mathrm{~K} / \mathrm{AKT} / \mathrm{mTOR}$ axis inhibitors. Mol Cancer Ther. 2011;10(3):558-565.

17. Weigelt B, Warne PH, Downward J. PIK3CA mutation, but not PTEN loss of function, determines the sensitivity of breast cancer cells to mTOR inhibitory drugs. Oncogene. 2011;30(29):3222-3233.

18. Dienstmann R, et al. Molecular profiling of patients with colorectal cancer and matched targeted therapy in phase I clinical trials. Mol Cancer Ther. 2012; 11(9):2062-2071.

19. Di Nicolantonio F, et al. Deregulation of the PI3K and KRAS signaling pathways in human cancer cells determines their response to everolimus. J Clin Invest. 2010;120(8):2858-2866.

20. Hung KE, et al. Development of a mouse model for sporadic and metastatic colon tumors and its use in assessing drug treatment. Proc Natl Acad SciUS A. 2010;107(4):1565-1570.

21. Muellner MK, et al. A chemical-genetic screen reveals a mechanism of resistance to PI3K inhibitors in cancer. Nat Chem Biol. 2011;7(11):787-793.
22. Ilic N, Utermark T, Widlund HR, Roberts TM. PI3K-targeted therapy can be evaded by gene amplification along the MYC-eukaryotic translation initiation factor 4E (eIF4E) axis. Proc Natl Acad SciUS A. 2011;108(37):E699-E708.

23 . Tenbaum SP, et al. $\beta$-Catenin confers resistance to $\mathrm{PI} 3 \mathrm{~K}$ and AKT inhibitors and subverts FOXO3a to promote metastasis in colon cancer. Nat Med. 2012; 18(6):892-901.

24. Liu P, et al. Oncogenic PIK3CA-driven mammary tumors frequently recur via PI3K pathway-dependent and PI3K pathway-independent mechanisms. Nat Med. 2011;17(9):1116-1120.

25. Anjum R, Blenis J. The RSK family of kinases: emerging roles in cellular signalling. Nat Rev $\mathrm{Mol}$ Cell Biol. 2008;9(10):747-758.

26. Romeo Y, Zhang X, Roux PP. Regulation and function of the RSK family of protein kinases. Biochem J. 2011;441(2):553-569.

27. Lopez-Vicente L, et al. Regulation of replicative and stress-induced senescence by RSK4, which is down-regulated in human tumors. Clin Cancer Res. 2009;15(14):4546-4553.

28. Lopez-Vicente L, et al. RSK4 inhibition results in bypass of stress-induced and oncogene-induced senescence. Carcinogenesis. 2011;32(4):470-476.

29. Maira SM, et al. Identification and characterization of NVP-BEZ235, a new orally available dual phosphatidylinositol 3-kinase/mammalian target of rapamycin inhibitor with potent in vivo antitumor activity. Mol Cancer Ther. 2008;7(7):1851-1863.

30. Maira SM, et al. Identification and characterization of NVP-BKM120, an orally available pan class I PI3-Kinase inhibitor. Mol Cancer Ther. 2012;11(2):317-328.

31. Bendell JC, et al. Phase I, dose-escalation study of BKM120, an oral pan-Class I PI3K inhibitor, in patients with advanced solid tumors. J Clin Oncol. 2012;30(3):282-290.

32. Yang $X$, et al. A public genome-scale lentiviral expres- 
sion library of human ORFs. Nat Methods. 2011; 8(8):659-661.

33. Boehm JS, Hahn WC. Towards systematic functional characterization of cancer genomes. Nat Rev Genet. 2011;12(7):487-498.

34. Johannessen CM, et al. COT drives resistance to RAF inhibition through MAP kinase pathway reactivation. Nature. 2010;468(7326):968-972.

35. Brachmann S, Fritsch C, Maira SM, Garcia-Echeverria C. PI3K and mTOR inhibitors: a new generation of targeted anticancer agents. Curr Opin Cell Biol. 2009;21(2):194-198.

36. Chandarlapaty S, et al. AKT inhibition relieves feedback suppression of receptor tyrosine kinase expression and activity. Cancer Cell. 2011;19(1):58-71.

37. Eichhorn PJ, et al. Phosphatidylinositol 3-kinase hyperactivation results in lapatinib resistance that is reversed by the $\mathrm{mTOR} / \mathrm{phosphatidylinositol}$ 3-kinase inhibitor NVP-BEZ235. Cancer Res. 2008; 68(22):9221-9230.

38. Serra V, et al. PI3K inhibition results in enhanced HER signaling and acquired ERK dependency in HER2-overexpressing breast cancer. Oncogene. 2011; 30(22):2547-2557.

39. Feldman ME, et al. Active-site inhibitors of mTOR target rapamycin-resistant outputs of mTORC1 and mTORC2. PLoS Biol. 2009;7(2):e38.

40. Sengupta S, Peterson TR, Sabatini DM. Regulation of the mTOR complex 1 pathway by nutrients, growth factors, and stress. Mol Cell. 2010;40(2):310-322.

41. Roux PP, et al. RAS/ERK signaling promotes site-specific ribosomal protein S6 phosphorylation via RSK and stimulates cap-dependent translation. J Biol Chem. 2007;282(19):14056-14064.

42. Silvera D, Formenti SC, Schneider RJ. Translational control in cancer. Nat Rev Cancer. 2010;10(4):254-266.

43. Ascierto PA, et al. Efficacy and safety of oral MEK162 in patients with locally advanced and unresectable or metastatic cutaneous melanoma harboring BRAFV600 or NRAS mutations. J Clin Oncol. 2012;30(suppl):abstr 8511.

44. Nguyen TL. Targeting RSK: an overview of small molecule inhibitors. Anticancer Agents Med Chem. 2008;8(7):710-716.

45. Bain J, et al. The selectivity of protein kinase inhibitors: a further update. Biochem J. 2007;408(3):297-315.

46. Yeh TC, et al. Biological characterization of ARRY142886 (AZD6244), a potent, highly selective mitogen-activated protein kinase kinase $1 / 2$ inhibitor.
Clin Cancer Res. 2007;13(5):1576-1583.

47. Ibrahim $\mathrm{YH}$, et al. PI3K inhibition impairs BRCA1/2 expression and sensitizes BRCA-proficient triple-negative breast cancer to PARP inhibition. Cancer Discov. 2012;2(11):1036-1047.

48. Hennessy BT, et al. A technical assessment of the utility of reverse phase protein arrays for the study of the functional proteome in non-microdissected human breast cancers. Clin Proteomics. 2011;6(4):129-151.

49. Hoeflich KP, et al. In vivo antitumor activity of MEK and phosphatidylinositol 3-kinase inhibitors in basal-like breast cancer models. Clin Cancer Res. 2009;15(14):4649-4664.

50. Uhlen M, et al. Towards a knowledge-based human protein atlas. Nat Biotechnol. 2010;28(12):1248-1250.

51. Barretina J, et al. The Cancer Cell Line Encyclopedia enables predictive modelling of anticancer drug sensitivity. Nature. 2012;483(7391):603-607.

52. Shahbazian D, et al. The mTOR/PI3K and MAPK pathways converge on eIF4B to control its phosphorylation and activity. EMBOJ. 2006;25(12):2781-2791.

53. Smolen GA, et al. A genome-wide RNAi screen identifies multiple RSK-dependent regulators of cell migration. Genes Dev. 2010;24(23):2654-2665.

54. Xing J, Ginty DD, Greenberg ME. Coupling of the RAS-MAPK pathway to gene activation by RSK2, a growth factor-regulated CREB kinase. Science. 1996;273(5277):959-963.

55. Bignone PA, et al. RPS6KA2, a putative tumour suppressor gene at $6 \mathrm{q} 27$ in sporadic epithelial ovarian cancer. Oncogene. 2007;26(5):683-700.

56. Clark DE, Errington TM, Smith JA, Frierson HF Jr, Weber MJ, Lannigan DA. The serine/threonine protein kinase, p90 ribosomal S6 kinase, is an important regulator of prostate cancer cell proliferation. Cancer Res. 2005;65(8):3108-3116.

57. Smith JA, Poteet-Smith CE, Xu Y, Errington TM, Hecht SM, Lannigan DA. Identification of the first specific inhibitor of $\mathrm{p} 90$ ribosomal S6 kinase (RSK) reveals an unexpected role for RSK in cancer cell proliferation. Cancer Res. 2005;65(3):1027-1034.

58. Berns $\mathrm{K}$, et al. A large-scale RNAi screen in human cells identifies new components of the p53 pathway. Nature. 2004;428(6981):431-437.

59. Lee S, et al. RSK-mediated phosphorylation in the C/EBP $\{$ beta\} leucine zipper regulates DNA binding, dimerization, and growth arrest activity. Mol Cell Biol. 2010;30(11):2621-2635.

60. Thakur A, et al. Aberrant expression of X-linked genes RbAp46, Rsk4, and Cldn2 in breast cancer. Mol Cancer Res. 2007;5(2):171-181.

61. Thakur A, et al. Anti-invasive and antimetastatic activities of ribosomal protein S6 kinase 4 in breast cancer cells. Clin Cancer Res. 2008;14(14):4427-4436.

62. Dummler BA, et al. Functional characterization of human RSK4, a new 90-kDa ribosomal S6 kinase, reveals constitutive activation in most cell types. J Biol Chem. 2005;280(14):13304-13314.

63. Bender C, Ullrich A. PRKX, TTBK2, and RSK4 expression causes Sunitinib resistance in kidney carcinoma- and melanoma-cell lines. Int J Cancer. 2012;131(2):E45-E55.

64. Lawson TG, et al. Dissociation of double-stranded polynucleotide helical structures by eukaryotic initiation factors, as revealed by a novel assay. Biochemistry. 1989;28(11):4729-4734.

65. Rozen F, Edery I, Meerovitch K, Dever TE, Merrick WC, Sonenberg N. Bidirectional RNA helicase activity of eucaryotic translation initiation factors 4A and 4F. Mol Cell Biol. 1990;10(3):1134-1144

66. Methot N, Song MS, Sonenberg N. A region rich in aspartic acid, arginine, tyrosine, and glycine (DRYG) mediates eukaryotic initiation factor 4B (eIF4B) self-association and interaction with eIF3. Mol Cell Biol. 1996;16(10):5328-5334.

67. Ilic N, Utermark T, Widlund HR, Roberts TM. PI3K-targeted therapy can be evaded by gene amplification along the MYC-eukaryotic translation initiation factor 4E (eIF4E) axis. Proc Natl Acad Sci US A. 2011;108(37):E699-E708.

68. Schatz JH, et al. Targeting cap-dependent translation blocks converging survival signals by AKT and PIM kinases in lymphoma. J Exp Med. 2011; 208(9):1799-1807.

69. Shapiro G, et al. Clinical combination of the MEK inhibitor GDC-0973 and the PI3K inhibitor GDC0941: A first-in-human phase Ib study testing daily and intermittent dosing schedules in patients with advanced solid tumors. J Clin Oncol. 2011; 29(suppl): abstr 3005.

70. Shimizu T, et al. The clinical effect of the dual-targeting strategy involving PI3K/AKT/mTOR and RAS/MEK/ERK pathways in patients with advanced cancer. Clin Cancer Res. 2012;18(8):2316-2325.

71. Gong J, Traganos F, Darzynkiewicz Z. A selective procedure for DNA extraction from apoptotic cells applicable for gel electrophoresis and flow cytometry. Anal Biochem. 1994;218(2):314-319. 